\title{
Prevalence of hypertension and metabolic disturbances in a population-based cohort of postmenopausal women. The Gniewkowo Women Study
}

\author{
Tadeusz Dereziński ${ }^{1}$, Jacek Wolf ${ }^{2,3}$, Beata Wąsikowska', Anna Szyndler ${ }^{2}$, Marzena Chrostowska², \\ Krzysztof Narkiewicz ${ }^{2,3}$ \\ ${ }^{1}$ Esculap Medical Centre, Gniewkowo, Poland \\ ${ }^{2}$ Department of Hypertension and Diabetology, Medical University of Gdansk, Gdansk, Poland \\ ${ }^{3}$ International Clinical Research Centre, St. Anne's University Hospital Brno, Brno, Czech Republic
}

\section{Summary}

Background Metabolic syndrome (MS) constitutes a major determinant of cardiovascular (CV) morbidity and mortality in adults. This phenomenon in women before menopause is attenuated by gonadal hormonal activity. After menopause the CV disease (CVD) incidence among women increases dramatically, and often overtakes the prevalence observed among men. Little is known about the CV-risk characteristic of early postmenopausal women in our country. Therefore the aim of our study was to investigate the frequency and distribution of metabolic syndrome components in early postmenopausal women residing urban-rural area in central Poland.

Material and Methods All women aged 65-75 years, opted for one general practice form an urban-rural area in Central Poland were invited to participate. The anthropometric measures, blood pressure and blood sampling were performed. For the purpose of the study, the modified NCEP ATP III criteria for the diagnosis of metabolic syndrome were used.

Results $59.5 \%$ of eligible women accepted the invitation. Additional $5.2 \%$ of randomly selected women from initially non-responders group were added to the investigated population. In the studied group only $15.7 \%$ had normal body weight and $82.4 \%$ met the criteria for abdominal obesity ( $92 \%$ according to IDF). $81.9 \%$ of women were characterized by hypertension, even more met the NCEP ATP III criterion for elevated blood pressure (BP) (91.5\%). Only $24.2 \%$ of studied women were treated effectively; moreover, despite treatment with three antihypertensive drugs $17.4 \%$ hypertensive women did not reach target BP values. Over one quarter of studied women suffered from diabetes mellitus, among whom $25.5 \%$ were not aware of the presence of the disease. Another $37.1 \%$ of women presented with prediabetic condition. Lipid disturbances were noted in the $25.3 \%$ and $18.4 \%$ of women for triglycerides (TG) increase and low HDL-C levels, respectively. According to modified NCEP ATP III criteria we were able to diagnose $58.8 \%$ of studied women with metabolic syndrome. Additionally, $31.9 \%$ of postmenopausal women were active cigarette smokers.

Conclusions Metabolic profile of early postmenopausal women in central Poland is strikingly abnormal. High incidence of metabolic syndrome is mainly determined by extremely prevalent increased blood pressure and abdominal obesity. With relation to all identified metabolic syndrome components, the strategy aimed at the improvement of body mass status, diabetes diagnosis and especially blood pressure control appear to be of highest priority.

key words: hypertension, metabolic syndrome, menopause, women

Arterial Hypertens. 2015, vol. 19, no. 2, pages: 88-94

DOI: $10.5603 / A H .2015 .0012$

Address for correspondence: Prof. Krzysztof Narkiewicz

Debinki str. 7C, 80-952 Gdansk, Poland, Tel. +48583492527, Fax: +48583492601

E-mail: knark@gumed.edu.pl

VM Copyright @ 2015 Via Medica, ISSN 2449-6170 


\section{Background}

Clustering of metabolic abnormalities commonly known as metabolic syndrome (MS) constitutes a major determinant of prevalent cardiovascular (CV) morbidity and mortality in adults [1]. However, this relationship in women before the menopause is markedly attenuated by gonadal hormonal activity. Therefore, over past decades a special emphasis was put on the exploration of the interrelationships between $\mathrm{CV}$ risk factors and $\mathrm{CV}$ consequences predominantly in men. Declining gonadal hormonal activity in women coincides with a dramatic increase in CV risk [2] which is usually underestimated by health-providers. Hypoestrogenism exert a negative impact on adipose tissue metabolism which along with physical inactivity favour central-body obesity development [2-4]. In turn, abdominal obesity via hormonal and molecular actions [5] is further implicated in the pathogenesis of hypertension, type 2 diabetes and dyslipidaemia. Consequently, women after menopause not only start to overtake men with different $\mathrm{CV}$ diseases but often $\mathrm{CV}$-morbidity becomes overrepresented as compared to their male-counterparts. One of the strongest determinants accountable for the observed increase in $\mathrm{CV}$ disease (CVD) in postmenopausal women may be exceedingly prevalent and poorly controlled hypertension. High blood pressure itself is a leading cause of cerebrovascular events [6] as well as the premature deaths in the westernized societies [7]. Except for some epidemiological aspects [8], little is known about the specific characteristic of early postmenopausal hypertension in women in our country. Data related to difficult-to-control or resistant hypertension in this group of patients are also sparse. Therefore we sought to investigate the frequency and the distribution of metabolic syndrome components in early postmenopausal women residing urban-rural area in central Poland with special emphasis put on blood pressure control.

\section{Material and methods}

To conduct the study we invited all women aged 65 to 75 years old who at time of the recruitment (2013) dwelled the urban-rural Gniewkowo County in Central Poland (urban-rural population approximately $50 \%$-to- $50 \%$ ), and had opted a family medical service. None of the health-related condition was an exclusion criterion from the study. Following the initial assessment of all responders, an additional weighted sample was recruited from the group of eligible women who did not accept primary invitation. In order to select a non-responder representatives we performed a sample size calculation based on the power analysis. The assumption for the power analysis was primary based on the effect of the BMI status as the body fat translates to complex metabolic disturbances commonly clustering in metabolic syndrome. Accordingly, additional group of randomly selected 33 women received repeated invitation, which was finally accepted by all of them.

The clinical assessment of study participants consisted of anthropometric measurements that is body weight, waist circumference, height, body mass index (BMI); office blood pressure measurements (average of the second and the third measurement according to ESH); blood sampling (responders only) i.e. lipid profile, fasting blood glycaemia, and oral glucose tolerance test in all cases where fasting plasma glucose levels were equal or greater than $5.5 \mathrm{mmol} / \mathrm{L}$ and the participant denied established diabetes. For the purpose of our study we incorporated the definition of metabolic syndrome according to modified NCEP ATP III criteria $[9,10]$. The presence of the three of the following criteria allowed for the MS diagnosis i.e. waist circumference $\geq 88 \mathrm{~cm}$, triglycerides levels $\geq 150 \mathrm{mg} / \mathrm{dL}(1.7 \mathrm{mmol} / \mathrm{L})$, reduced HDL-C less than $50 \mathrm{mg} / \mathrm{dL}(1.3 \mathrm{mmol} / \mathrm{dL})$, elevated $\mathrm{BP} \geq$ $130 \mathrm{~mm} \mathrm{Hg}$ for systolic and/or $\geq 85 \mathrm{~mm} \mathrm{Hg}$ of diastolic blood pressure, and elevated fasting plasma glucose above $100 \mathrm{mg} / \mathrm{dL}(5.5 \mathrm{mmol} / \mathrm{L})$ or established diabetes mellitus. All participants gave written consent for data collection and analyses.

\section{Statistical analysis}

All data were tabulated in MS Excel and computed in Statistica 10, StatSoft Inc. (licenced to Medical University of Gdansk). Data were averaged and presented as means $\pm \mathrm{SD}$ and/or medians (IQR), if appropriate. Between-group comparisons were performed using unpaired t-test or Mann-Whitney tests, where appropriate. The prevalence of particular disorders was presented as per cent. Power analysis was performed to estimate a sample selected from a non-responder group. P-value less than 0.05 was considered valid for all calculations.

\section{Results}

A total of 364 participants were initially enrolled in the study (59.5\% response-rate) which together with additional randomly selected 33 women accounted for $62.6 \%$ of a total eligible population. The clinical characteristic of the study group is pre- 
Table I. Clinical characteristic of the study group $(n=364)$

\begin{tabular}{l|c}
\hline Age (years) & $69.2 \pm 3.3$ \\
\hline Weight (KG) & $76.1 \pm 13.6$ \\
\hline BMl [KG/m²] & $30.7 \pm 5.3$ \\
\hline Waist [cm] & $98.0 \pm 12.4$ \\
\hline Total Cholesterol [mg/dl] & $214.5 \pm 49.0$ \\
\hline LDL [mg/dl] & $124.2 \pm 45.0$ \\
\hline HDL [mg/dl] & $65.9 \pm 17.6$ \\
\hline Triglycerides [mg/dl] & $127.4 \pm 65.0$ \\
\hline Glucose [mg/dl] & $109.9 \pm 28.3$ \\
\hline Serum creatinine [mg/dl] & $0.7 \pm 0.2$ \\
\hline eGFR (MDRD) & $83.7 \pm 23.4$ \\
\hline eGFR (Cockcroft-Gault) & $90.1 \pm 24.8$ \\
\hline eGFR (EPI) & $82.0 \pm 15.9$ \\
\hline Systolic BP [mm Hg] & $150.2 \pm 22.1$ \\
\hline Diastolic BP [mm Hg] & $83.7 \pm 10.4$ \\
\hline No. of antihypertensive drugs & $1.7 \pm 1.4$ \\
\hline No. of MS components & $3.1 \pm 1.1$ \\
\hline
\end{tabular}

BMI — body mass index; LDL — low density lipoprotein; HDL — high density lipoprotein; eGFR - estimated glomerular filtration rate; MDRD - Modification of Diet in Renal Disease equation; MS - metabolic syndrome

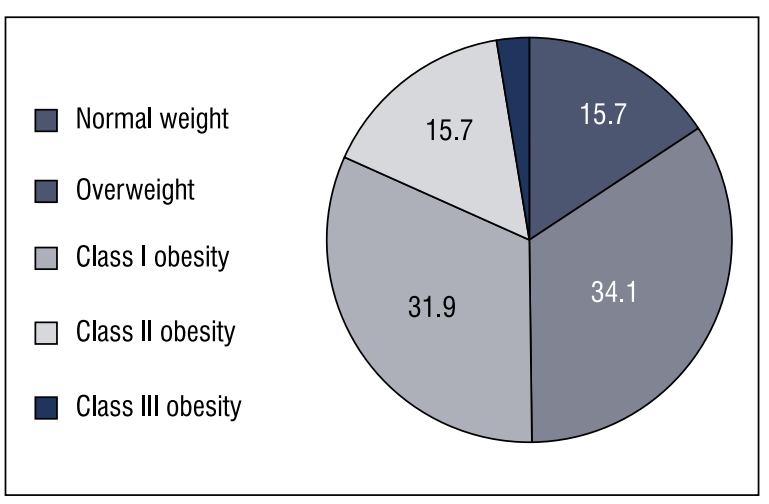

Figure 1. BMI status

sented in Table I. Neither BMI nor blood pressure differed significantly in non-responder vs. responder group.

Based on the BMI analysis, only $15.7 \%$ of women had a normal body weight and vast majority $(82.4 \%)$ met the NCEP ATP III criteria for the diagnosis of abdominal obesity ( $92 \%$ according to IDF). Detailed BMI distribution is presented in Figure 1.

Similar fraction of studied women $(81.9 \%)$ was characterized by hypertension i.e. either de novo $(13.1 \%)$ or established hypertension. Even more met the NCEP ATP III criterion for elevated blood pressure $(91.5 \%)$. Although the average duration of

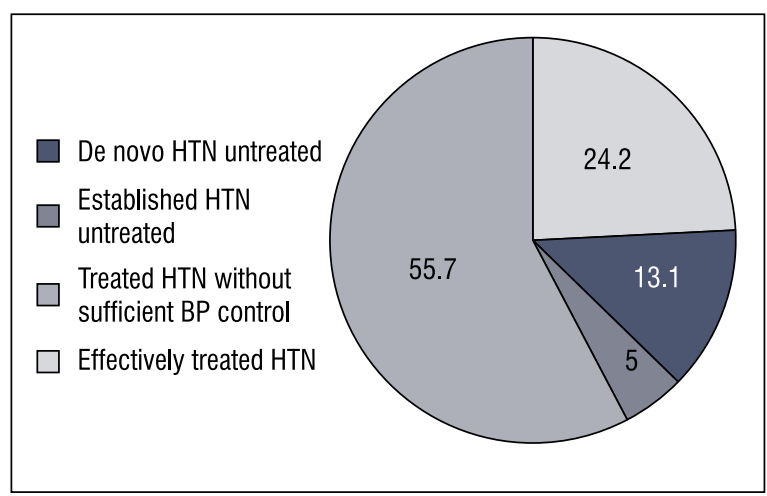

Figure 2. Blood-lowering treatment efficacy in the complete studied population of the postmenopausal women (both aware and unaware of hypertension). Data presented as per cent HTN — hypertension; BP — blood pressure

hypertensive disease was $11.4 \pm 9.2$ years, women who were aware of the diagnosed hypertension were treated effectively only in $24.2 \%$ (Figure 2). The distribution of the number of antihypertensive drugs was as follows: none $-27 \%$, one drug $-19 \%$, two drugs - $27 \%$, three drugs - $16 \%$, four drugs $10 \%$, and five drugs - $2 \%$. Specific percentage of blood-lowering drugs usage is presented in Figure 3. A fraction of $17.4 \%$ hypertensive women did not reach target blood pressure values of $<140 \mathrm{~mm} \mathrm{Hg}$ for systolic and/or $<90 \mathrm{~mm} \mathrm{Hg}$ for diastolic levels despite treatment with three anti-hypertensive medicines including a diuretic. The mean values of systolic blood pressure were tightly related to quantity of clustering metabolic syndrome components (Figure 4).

A considerable number of studied women (25.8\%) suffered from diabetes mellitus, among whom $25.5 \%$ were not aware of the presence of the disease (newly diagnosed cases accounted for $6.6 \%$ of the studied population). Another $37.1 \%$ of women presented with one or two of the prediabetic condition - Figure 5.

The total cholesterol (TC) in statin-treated women equalled $196 \pm 48.6$, and LDL-C $=107.3 \pm 46.0$ which was significantly lower comparing to the results seen in untreated women: $226.3 \pm 44.2$, and $137.9 \pm 39.2$ for TC and LDL-C, respectively. However, the HDL-C and triglycerides (TG) were comparable in both groups $66.3 \pm 17.4$ vs. $65.5 \pm 17.9$, $\mathrm{P}=0.66$; and $130.0 \pm 69.3$ vs. $124.1 \pm 59.2, \mathrm{P}=0.39$ for untreated vs. treated with lipid-lowering agents, respectively.

The prevalence of metabolic syndrome and the distribution of its components are presented in Figure 6 and Figure 7.

Additionally, $31.9 \%$ of postmenopausal women were active cigarette smokers. 


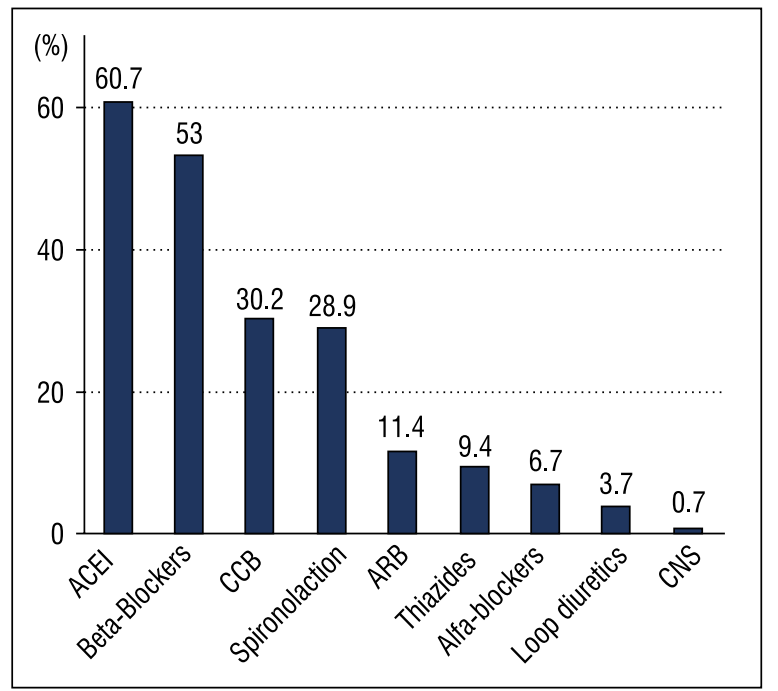

Figure 3. Antihypertensive drugs distribution

ACEI — angiotensin converting enzyme inhibitors; $C \mathrm{CB}$ - calcium channel blockers; ARB — angiotensin receptor blockers; CNS — central nervous system

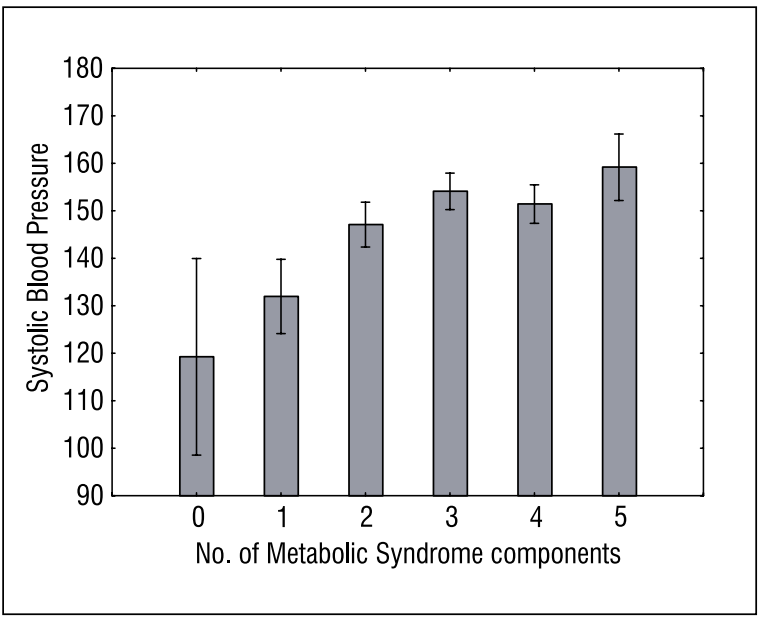

Figure 4. Relationship of the number of antihypertensive drugs with systolic blood pressure values. One-way ANOVA, P $<0.001$

\section{Discussion}

The main message from our study is that metabolic syndrome is extremely prevalent in early postmenopausal years in an urban-rural female population in central Poland. Evidently, high blood pressure and abdominal obesity are the main determinants of this phenomenon.

Body fat excess, especially with its abdominal distribution together with low physical activity constitute main causative factors for the increased insulin resistance followed by the development of metabolic syndrome [11]. Clustering of metabolic abnormalities has in turn deleterious influence on cardiovascular profile, and significantly determines increased morbidity and mortality in westernized societies.

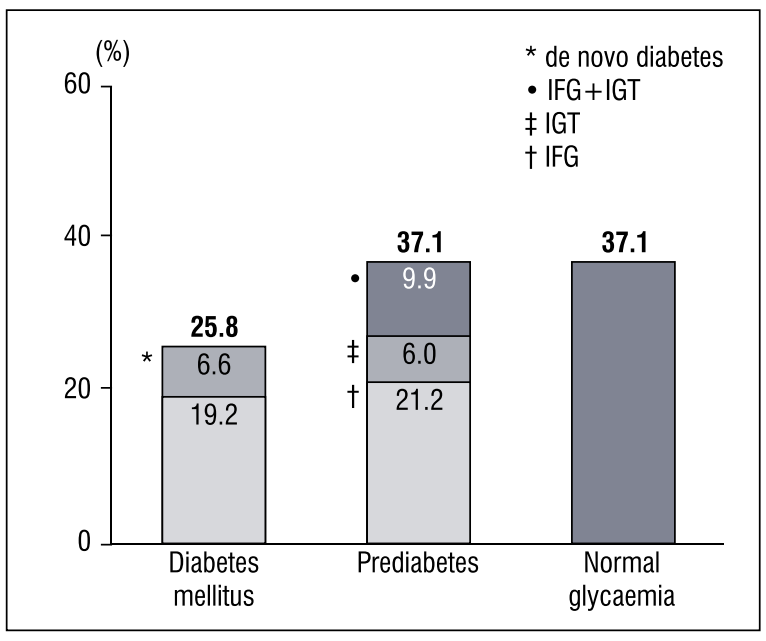

Figure 5. Glycaemia control

IFG — impaired fasting glucose; IGT — Impaired glucose tolerance

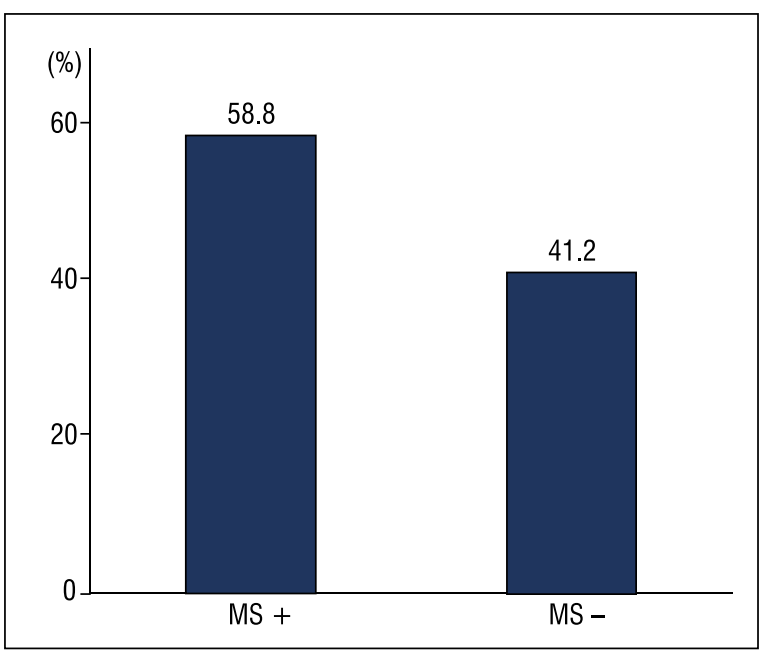

Figure 6. Prevalence of Metabolic Syndrome in women aged 65to-75 years

MS - Metabolic Syndrome

A meta-analysis of data exploring the impact of MS on cardiovascular system which included a total of 172,573 patients showed that MS increases the risk of cardiovascular events by 1.78 -fold, and this relationship is even more evident in female patients ( RR $=2.63)[12]$.

In the procreative age, cardiac saving-effect ascribed to female sex-hormones determines the gap in the incidence of cardiovascular disease reported in both genders. Although women start to overtake men with different $\mathrm{CV}$ diseases after menopause [13]; this phenomenon may be only partially explained by gonadal function decline. Our cross-sectional study clearly shows that soon after the onset of their menopause majority of women (58.8\%) 


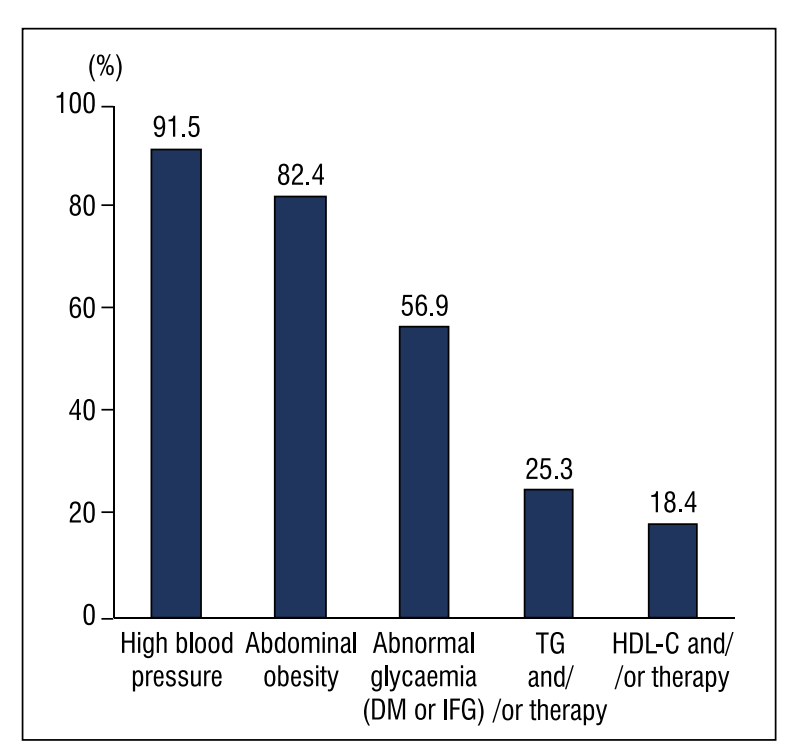

Figure 7. Metabolic Syndrome components frequency distribution in postmenopausal women (age range from 65 to 75 years). Data presented as per cent

meet the criteria of metabolic syndrome. It is thus justified to hypothesize that MS constitutes one of the substantial negative determinants accountable for increased cardiovascular disease rate and premature deaths seen in postmenopausal women in Poland. In addition, there is an alarming trend recorded in the prevalence of MS in women. The WOBASZ study data which were published 10 years ago [14] documented $46.3 \%$ of incident MS in women aged $60-75$ years. The discrepant results between WOBASZ study and our analysis $(46.3 \%$ vs. $58.8 \%$; respectively) may result from several factors. Five years of difference for the entry criteria may be partially accounted for the observed difference, since WOBASZ Study clearly showed stepwise increase in the incident MS in women across different age-subgroups. Second, as reported in WOBASZ Study, there are marked differences in the prevalence of MS crosswise voivodeships of Poland. Accordingly, the voivodeship we studied was one with the highest MS rate seen in 2005 .

Third, and probably the most important, is the fact that the epidemiology of metabolic disturbances shows growing number over past decades in Poland. Recently announced data from NatPOL 2011 Study documented slightly higher prevalence of MS in women in comparable age (60 to 79 years) as we report now (63\% vs. $58.8 \%$, respectively), which contrasts with the data from WOBASZ Study 2005 $(46.3 \%)$. Most probable scenario is that one of the main determinants of the development of MS is the growing epidemic of abdominal obesity [15].

\section{Blood pressure}

In the studied population, approximately $18 \%$ of females were not aware of having the indication to blood-lowering therapy (13.1\% of newly diagnosed hypertension and $5 \%$ not treated), and vast majority $(57.7 \%)$ who were on current treatment did not reach target blood pressure values. However, our data show a comparable percentage of effectively treated patients as reported in NatPOL Study in 2011 (both sexes) i.e. $24.2 \%$ vs. $26 \%$, respectively. It is not clear why only a fraction of $24.2 \%$ of women in postmenopausal age with established hypertension is characterized by satisfactory blood pressure control regardless of multiple and modern antihypertensive drug regimens (Figure 2, 3). In addition, as many as $17.4 \%$ of postmenopausal hypertensive female patients do not reach target blood pressure levels irrespective of three blood-lowering medicines, including diuretics. The difficulties with hypertension control in this population raise a valid question about a real cause of this phenomenon. The design of our observational study did not allow us to verify the actual reason of unsatisfactory BP levels, however, the poor adherence to prescribed treatment regimen probably explains only part of the problem. It is also unlikely that secondary causes of hypertension account for such a poor outcome. In general, the mechanisms why women after the menopause have relatively higher incident hypertension comparing to men [16] is not well understood, especially that women are more likely to adhere to therapeutic regimens. National Statistics in the United Stated documented that more than $75 \%$ of women aged 60 years old or older become hypertensive [13]. One may speculate that extremely high metabolic syndrome incidence $(58.8 \%)$ via complex mechanisms significantly enhance blood pressure levels. Figure 5 depicts an evident interdependence between the increasing systolic blood pressure values and the number of clustering metabolic syndrome components. A reasonable explanation for these relationships may be extrapolated from studies in obstructive sleep apnoea (OSA). OSA has been attributed to abnormal diurnal blood pressure control, increasing odds for hypertension development, and difficulties in blood pressure lowering with pharmacological treatment. Since obesity is the major risk factor for the development of OSA, the concurrence of sleep disordered breathing and metabolic disturbances is very common. In fact, it is estimated that metabolic disturbances are approximately 9-times more likely to be present in subjects with OSA [17]. This hypothesis may be further supported by the observation that women after procreative age are more likely to deve- 
lop a nondipping pattern of diurnal BP [18] comparing to their male counterparts. Such abnormal blood pressure profile is a common characteristic in sleep apnoea [19].

\section{Glycaemia control}

International Diabetes Federation estimates that patients with MS are characterized by 5 -fold increased risk for the development of type 2 diabetes, a condition which negatively influences estimated life-span and its quality. In our study, $25.8 \%$ of postmenopausal women had already established type 2 diabetes diagnosis, among whom $25.5 \%$ were not aware of this burden. This result fairly corresponds with prevalent type 2 diabetes mellitus recently reported by Authors of NatPOL 2011 [20] (25-30\% in subjects aged $>65$ years). As a significant fraction of all diabetic women from Gniewkowo-County were not aware of the presence of the disease $(25.5 \%)$, it appears that the implementation of systematic screening programs is critical in order to better identify the population at high and/or very high cardiovascular risk according to risk stratification strategies [21]. Another $37.1 \%$ of the postmenopausal women met the criteria of the prediabetic state (IFG and/ /or IGT). Taking into account the phenotype of the studied group $(82.4 \%$ with abdominal obesity), one may speculate that a considerable fraction of prediabetic women will further progress to overt type 2 diabetes mellitus, unless prompt and complex preventive measures are introduced to stop this modifiable sequel.

\section{Lipids}

The analysis of lipid profiles in dichotomized subgroups documented clinically relevant net difference in total cholesterol and LDL-C levels in postmenopausal female population, which may be accounted for the common use of statins. In our sample, $44.4 \%$ of women were administered with statins, which is considerably higher than recently reported by NatPOL Investigators (32\% in subjects aged 60-79 years) [22]. Consequently, widespread treatment with lipid-lowering drugs allowed to reach the reasonable mean level of LDL-C equal $107.3 \pm 46.0$ which is close to target LDL-C levels $<100 \mathrm{mg} / \mathrm{dL}$ $(2.5 \mathrm{mmol} / \mathrm{dL})$ recommended in patients at high cardiovascular risk (excluding diabetics and CV complicated patients [23]). The residual risk, which is partially ascribed to elevated triglycerides and low HDL-C levels, appears to be less important than hypercholesterolaemia in this population. Mean value of TG was $127.4 \pm 65.0$ (target $<150 \mathrm{mg} / \mathrm{dL}$ ) and HDL-C $=66 \mathrm{mg} / \mathrm{dL}$ for in the whole group of postmenopausal women (Table 1). Nonetheless, it is worth underlining that a fraction of approximately $25 \%$ and $18 \%$ met the criteria of hypertriglyceridaemia and/or low HDL-C, respectively.

\section{Study limitations}

The clinical data presented in our study are derived from the population who already had health-related records in Gniewkowo County; therefore no information from women who did not opt primary care was available. It is possible that women who have never sought any sort of medical assistance are by definition healthier; however, from the statistical standpoint, one may assume that the distribution of subjects who are healthy vs. non-healthy etc. is balanced. Additionally, the efficacy of the recruitment (final response-rate at $62.6 \%$ ) closely reflects the ratio achieved in the largest epidemiological studies completed in Poland (NatPOL 2011 response-rate at $66.4 \%$ [24]).

\section{Conclusions}

Metabolic profile of early postmenopausal women dwelling in central Poland is strikingly abnormal. High incidence of metabolic syndrome is mainly determined by extremely prevalent hypertension and abdominal obesity. With relation to all identified metabolic syndrome components, the strategy aimed at the improvement of body mass status, diabetes diagnosis and especially blood pressure control appear to be of highest priority in postmenopausal women in Poland.

\section{References}

1. Isomma B., Almgren P., Tuomi T. et al. Cardiovascular mortality and morbidity associated with the metabolic syndrome. Diabetes Care 2001; 24: 683-689.

2. Sowers M., Zheng H., Tomey K. et al. Changes in body composition in women over six years at midlife: ovarian and chronological aging. J. Clin. Endocrinol. Metab. 2007; 92: 895-901.

3. Douchi T., Kosha S., Uto H. et al. Precedence of bone loss over changes in body composition and body fat distribution within a few years after menopause. Maturitas 2003; 46: 133-138.

4. Wang Y.C., Colditz G.A., Kuntz K.M. Forecasting the obesity epidemic in the aging US. Popul. Obes. (Silver Spring) 2007; 15: 2855-2865.

5. Kershaw E.E., Flier J.S. Adiposse fat tissue as an endocrine organ. J. Clin. Endocrinol. Metab. 2004; 89: 2548-2556.

6. Mancia G., Fagard R., Narkiewicz K. et al. for ESH/ESC Task Force for the Management of Arterial Hypertension. 2013 Practice guidelines for the management of arterial hypertension of the European Society of Hypertension (ESH) and the European Society of Cardiology (ESC): ESH/ESC Task Force for the Management of Arterial Hypertension. J. Hypertens. 2013; 31: 1925-1938.

7. The world health report 2002: reducing risks, promoting healthy life. Geneva, World Health Organization, 2002. 
8. Chrostowska M., Szyndler A., Paczwa P., Narkiewicz K. Impact of abdominal obesity on the frequency of hypertension and cardiovascular disease in Poland - results from the IDEA study (International day for the evaluation of abdominal obesity). Blood Press. 2011; 20: $145-152$.

9. Expert Panel on Detection Evaluation, and Treatment of High Blood Cholesterol in Adults. Executive Summary of the Third Report of the National Cholesterol Education Program (NCEP) Expert Panel on Detection, Evaluation and Treatment of High Blood Cholesterol in Adults (Adult Treatment Panel III). JAMA 2001; 285: 2486-2497.

10. Grundy S.M., Cleeman J.I., Daniels S.R. et al. American Heart Association; National Heart, Lung, and Blood Institute. Diagnosis and management of the metabolic syndrome: an American Heart Association/National Heart, Lung, and Blood Institute Scientific Statement. Circulation 2005; 112: 2735-2752.

11. Grundy S.M. Metabolic syndrome: a multiplex cardiovascular risk factor. J. Clin. Endocrinol. Metab. 2007; 92: 399-404.

12. Gami A.S., Witt B.J., Howard D.E. et al. Metabolic syndrome and risk of incident cardiovascular events and death: a systematic review and meta-analysis of longitudinal studies. J. Am. Coll. Cardiol. 2007; 49: 403-414.

13. Lima R., Wofford M., Reckelhoff J.F. Hypertension in Postmenopausal Women. Curr. Hypertens. Rep. 2012; 14: 254-260.

14. Wyrzykowski B., Zdrojewski T., Sygnowska E. et al. Epidemiologia zespołu metabolicznego w Polsce. Wyniki programu WOBASZ. Kardiologia Polska 2005; 63: 6 (supl. 4).

15. Han T.S., Williams K., Sattar N., Hunt K.J., Lean M.E., Haffner S.M. Analysis of obesity and hyperinsulinemia in the development of metabolic syndrome: San Antonio Heart Study. Obes. Res. 2002; 10: $923-931$
16. Ong K.L., Tso A.W.K., Lam K.S., Cheung B.M. Gender differences in BP control and cardiovascular risk factors in Americans with diagnosed hypertension. Hypertension 2008; 51: 1142-1148.

17. Coughlin S.R., Mawdsley L., Mugarza J.A., Calverley P.M., Wilding J.P. Obstructive sleep apnoea is independently associated with an increased prevalence of metabolic syndrome. Eur. Heart J. 2004; 25: 735-741.

18. Routledge F.S., McFetridge-Durdle J.A., Dean C.R. Stress, menopausal status and nocturnal BP dipping patterns among hypertensive women. Can. J. Cardiol. 2009; 25: e157-163.

19. Davies C.W., Crosby J.H., Mullins R.L., Barbour C., Davies R.J., Stradling J.R. Case-control study of 24 hour ambulatory blood pressure in patients with obstructive sleep apnoea and normal matched control subjects. Thorax 2000; 55: 736-740.

20. Rutkowski M., Bandosz P., Czupryniak L. et al. Prevalence of diabetes and impaired fasting glucose in Poland: the NATPOL 2011 study. Diabet. Med. 2014; 31: 1568-1571.

21. Widecka K., Grodzicki T., Narkiewicz K. et al. Zasady postępowania w nadciśnieniu tętniczym — 2011 rok. Arterial Hypertension 2011; 15: 55-82.

22. Bandosz P., O'Flaherty M., Rutkowski M. et al. A victory for statins or a defeat for diet policies? Cholesterol falls in Poland in the past decade: a modeling study. Int. J. Cardiol. 2015; 185: 313-319.

23. Wożakowska-Kapłon B., Filipiak K.J., Mamcarz A. et al. Actual problems of dyslipidaemia treatment in Poland - 2nd Declaration of Sopot. Experts' Group Consensus endorsed by the Polish Cardiac Society Working Group on Cardiovascular Pharmacotherapy. Polish Heart Journal 2014; 72: 847-853.

24. Zdrojewski T., Rutkowski M., Bandosz P. et al. Prevalence and control of cardiovascular risk factors in Poland. Assumptions and objectives of the NATPOL 2011 Survey. Kardiol. Pol. 2013; 71: 381-392. 